\title{
Blind exploration of the unreferenced transcriptome reveals novel RNAs for
}

\section{prostate cancer diagnosis}

\section{Authors:}

M. Pinskaya ${ }^{\dagger}{ }^{\dagger}$, Z. Saci $^{1 \ddagger}$, M. Gallopin², N. H. Nguyen 2,3 , M. Gabriel${ }^{1}$, V. Firlej ${ }^{4,5}$, M.

Descrimes $^{1}$, A. de la Taille ${ }^{4,5,6}$, A. Londoño-Vallejo ${ }^{7}$, Y. Allory ${ }^{8}$, D. Gautheret ${ }^{2}$, A. Morillon ${ }^{1 *}$

\section{Affiliations:}

${ }^{1}$ ncRNA, epigenetic and genome fluidity, Université PSL, Sorbonne Université, CNRS, Institut Curie, Research Center, 26 rue d'Ulm, 75248 Paris, France

${ }^{2}$ Institute for Integrative Biology of the Cell, CEA, CNRS, Université Paris-Sud, Université Paris-Saclay, 91190 Gif sur Yvette, France

${ }^{3}$ Thuyloi University, 175 Tay Son, Dong Da, Hanoi, Vietnam

${ }^{4}$ Université Paris-Est, UPEC, F-94000 Créteil, France

5 INSERM, U955, Equipe 7, F-94000 Créteil, France

${ }^{6}$ AP-HP, Hôpital Henri Mondor, Département d’Urologie, Créteil, F-94000, France

7 Telomeres and cancer, Université PSL, Sorbonne Université, CNRS, Institut Curie, Research Center, 26 rue d'Ulm, 75248 Paris, France

${ }^{8}$ Compartimentation et dynamique cellulaire, Université PSL, Sorbonne Université, CNRS, Institut Curie, Research Center, 26 rue d'Ulm, 75248 Paris, France

${ }^{\dagger}$ equal contribution

${ }^{\ddagger}$ present address: Institute for Research in Immunology and Cancer, Université de Montréal, H3T 1J4, Canada, zohra.saci@umontreal.ca

*Correspondence to: Antonin Morillon, antonin.morillon@curie.fr; Daniel Gautheret, daniel.gautheret@u-psud.fr.

\section{Running title}

Blind discovery of RNA biomarkers

\section{Keywords}


bioRxiv preprint doi: https://doi.org/10.1101/644104; this version posted May 21, 2019. The copyright holder for this preprint (which was

not certified by peer review) is the author/funder, who has granted bioRxiv a license to display the preprint in perpetuity. It is made available under aCC-BY-NC-ND 4.0 International license.

RNA, biomarker, prostate cancer, differential expression, total RNA-sequencing, long noncoding RNA, predictive modeling 


\section{Abstract}

The broad use of RNA-sequencing technologies held a promise of improved diagnostic tools based on comprehensive transcript sets. However, mining human transcriptome data for disease biomarkers in clinical specimens is restricted by the limited power of conventional reference-based protocols relying on uniquely mapped reads and transcript annotations. Here, we implemented a blind reference-free computational protocol, DE-kupl, to directly infer RNA variations of any origin, including yet unreferenced RNAs, from high coverage total stranded RNA-sequencing datasets of tissue origin. As a bench test, this protocol was powered for detection of RNA subsequences embedded into unannotated putative long noncoding (lnc)RNAs expressed in prostate cancer tissues. Through filtering and visual inspection of 1,179 candidates, we defined 21 lncRNA probes that were further validated for robust tumorspecific expression by NanoString single molecule-based RNA measurements in 144 tissue specimens. Predictive modeling yielded a restricted probe panel enabling over $90 \%$ of true positive detection of cancer in an independent dataset from The Cancer Genome Atlas. Remarkably, this clinical signature made of only 9 unannotated lncRNAs largely outperformed PCA3, the only RNA biomarker approved by the Food and Drug Administration agency, specifically, in detection of high-risk prostate tumors. The proposed reference-free computational workflow is modular, highly sensitive and robust and can be applied to any pathology and any clinical application. 


\section{INTRODUCTION}

RNA sequencing (RNA-seq) has revolutionized our knowledge of human transcriptome and has been implemented as a pivot technique in clinical applications for the discovery of RNA-based biomarkers allowing disease diagnosis, prognosis and therapy follow-up. However, most biomarker discovery pipelines are blind to uncharacterized RNA molecules since they rely on the alignment of uniquely mapped reads to annotated references of the human transcriptome which are far from complete (UszczynskaRatajczak et al. 2018), (Deveson et al. 2018), (Morillon and Gautheret 2019). Indeed, state-specific unspliced variants, rare mRNA isoforms, RNA hybrids originating from trans-splicing or genome rearrangements, unannotated intergenic or antisense noncoding RNAs, mobile elements or viral genome insertions would be systematically missed. A recent approach to RNA-seq data analysis, DE-kupl, combines k-mer (subsequences of fixed size) decomposition and differential expression analysis to discover transcript variations yet unreferenced in the human transcriptome (Audoux et al. 2017). Applied to poly(A)+ RNA-seq datasets of in vitro cell system, DE-kupl unveiled a large number of RNA subsequences embedded into novel long noncoding RNAs. These transcripts of more than 200 nucleotides in length transcribed by RNA polymerase II from intergenic, intronic or antisense noncoding genomic locations constitute a prevalent class of human genes. Some lncRNAs are now recognized as precisely regulated stand-alone molecules participating in the control of fundamental cellular processes (Jarroux et al. 2017), (Quinn and Chang 2015). They show aberrant and specific expression in various cancers and other diseases promoting them as biomarkers, therapeutic molecules and drug targets (Leucci 2018), (Van Grembergen et al. 2016). Importantly, some lncRNAs can be robustly detected in biological fluids (blood and urine) as circulating molecules or encapsulated into extracellular vesicles, hence, raising an attractive possibility of IncRNA biomarkers usage in non-invasive clinical tests (Silva et al. 2015), (Deng et al. 2017), (Wang et al. 2014), (Zhao et al. 2018a), 
(Wang et al. 2018). The only example of an RNA-based biomarker so far introduced in clinical practice is the PCA3 IncRNA in prostate cancer (PCa) (de Kok et al. 2002). PCA3 is transcribed antisense to the tumor suppressor PRUNE2 gene and promotes its premessenger RNA editing and degradation (Salameh et al. 2015). Being overexpressed in 95\% of PCa cases, PCA3 is detected in urines and helps diagnosis providing, in addition to other clinical tests, more accurate metrics regarding repeated biopsies (Groskopf et al. 2006), (Galasso et al. 2010). However, it remains inaccurate in discrimination between low- and high-risk tumors since its expression may dramatically decrease in aggressive PCa cases tempering its systematic usage (Loeb and Partin 2011), (Alshalalfa et al. 2017).

Since PCA3 discovery and the development of RNA-seq technologies, the PCa transcriptome has been extensively explored by The Cancer Genome Atlas (TCGA) consortium and others to identify numerous PCa-associated lncRNAs (PCAT family) such as PCAT1, PCAT7 or PCAT114/SChLAP1 (Iyer et al. 2015), (Prensner et al. 2014). However, none of them has been yet introduced into clinical practice because of the variable expression incidence, as for SChLAP1 detected in 25\% of PCa cases presenting metastatic traits (Prensner et al. 2013), or low specificity, as PCAT1 or PCAT7, thus infringing their clinical value. Additional efforts are required for more accurate and exhaustive RNA identification, as well as more rigorous validations of clinical potency through independent RNA measurement technologies and clinical cohorts. Regardless a large number of transcriptomic studies and variety of clinical samples analyzed, discovery of RNA-based molecular biomarkers from publicly available RNA-seq datasets is still limited at two levels: (i) most experimental setups are based on poly(A) selected, unstranded cDNA sequencing, and (ii) computational analyses are generally focused on annotated genes and full-length RNA assemblies. This impedes the detection of low and poorly polyadenylated RNAs but also partially degraded RNAs from formalin-fixed paraffin-embedded (FFPE) tissues or other clinical samples (Zhao et al. 2018b), (Zhao et 
al. 2014). In addition, RNA-seq reads counting is less accurate at 5' RNA ends or even impossible for co-expressed paired sense/antisense transcripts and for yet unannotated RNAs among noncoding, fusion, repeat-derived transcripts (Audoux et al. 2017), (Davila et al. 2016).

Here, we propose a conceptually novel exploratory framework combining the total stranded RNA-seq of clinical samples and the reference-free DE-kupl algorithm for discovery of novel tumor-specific transcript variations. As a proof-of-concept, we focused on the least explored, noncoding portion of the genome devoid of annotated protein-coding sequences to build an exhaustive catalog of PCa associated subsequences (contigs) embedded into lncRNA genes. The catalog was further refined through minimal filtering to isolate the most potent subset of contigs and validate 21 of them by an alternative NanoString assay in the extended cohort of 144 prostate specimens. From this, a predictive modeling derived a panel of 9 yet unannotated lncRNAs validated for robust expression in an independent TCGA cohort. Importantly, its clinical performance surpassed the PCA3 lncRNA specifically in discrimination of high-risk tumors. The proposed probe-set can be further used for development of a PCa diagnostic test. Moving beyond this point, the proposed computational and experimental platform may serve as a tool for biomarkers discovery for any disease and any clinical task aiming at improved medical care and development of precision medicine approaches.

\section{RESULTS}

\section{Identification of PCa-specific RNA variants in the Discovery Set by DE-kupl}

The biomarker discovery workflow included three major phases: discovery, selection and validation (Fig. 1). First, for discovery, we performed a deep total stranded RNA-seq of ribosomal RNA-depleted RNA samples isolated from prostate tissues after radical prostatectomy (Discovery Set, PAIR cohort, Supplemental Table S1). This Discovery set was processed by DE-kupl to identify tumor-specific transcripts. DE-kupl directly queries FASTQ files for subsequences (k-mers) with differential counts/expression (DE) 
between two conditions (Fig. 2A) (Audoux et al. 2017). Overlapping k-mers are then assembled into contigs and, in a final step, mapped to the human genome for annotation. In the aim to focus exclusively on novel, yet unannotated RNA elements, k-mers exactly matching GENCODE annotated transcripts were masked. We eventually retained contigs longer than 200 nucleotides and showing adjusted p-values below 0.01 to capture the most significant expression changes linked either to new transcriptional or processing events within known or putative lncRNA loci.

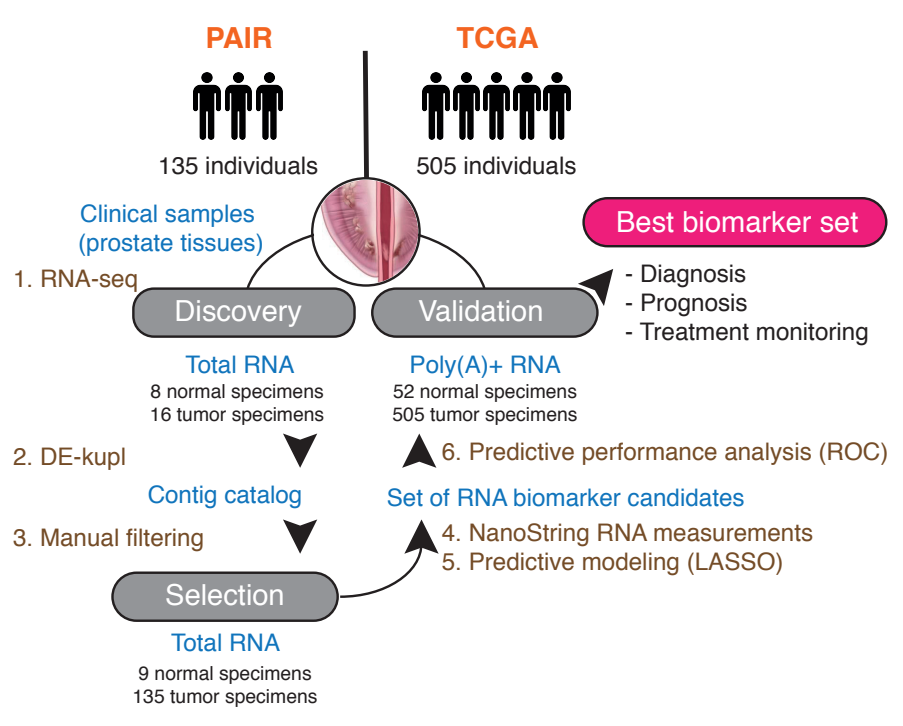

Figure 1. Experimental and computational workflow for discovery and validation of RNA-based clinical biomarkers. Raw total stranded RNA-seq data of a small clinical cohort is processed by DE-kupl to allow comparison of 8 normal against 16 tumor specimens (in this case formaldehyde fixed paraffin embedded tissues from radical prostatectomy) and cataloguing of all differentially expressed RNA variations (contigs). The whole set is filtered according to desired criteria and the top ranked contigs are selected for an independent experimental validation by NanoString in the extended clinical cohort. Finally, predictive modeling infers the best panel of candidate RNAs for validation of its clinical potency in an independent cohort (in this case TCGA).

With these criteria, we identified 1,179 tumor up-regulated contigs assigned to four main categories according to their mapping features: contiguous (uniquely mapped) contigs $(\mathrm{N}=935)$, splice variants $(\mathrm{N}=54)$, repeats $(\mathrm{N}=167)$ and unmapped contigs $(\mathrm{N}=23)$ (Fig. 2B, Fig. S1). Among them, 33.93\% and 6.36\% were embedded into already referenced GENCODE or MiTranscriptome IncRNA genes, respectively, but represented new sequence variations or RNA processing events, as PCAT7 (ctg_111348, P16) or 
bioRxiv preprint doi: https://doi.org/10.1101/644104; this version posted May 21, 2019. The copyright holder for this preprint (which was

not certified by peer review) is the author/funder, who has granted bioRxiv a license to display the preprint in perpetuity. It is made available under aCC-BY-NC-ND 4.0 International license.

CTBP1-AS (ctg_25348, P10). The rest mapped to intergenic noncoding locations or antisense to referenced protein-coding or noncoding genes (Fig. 2C). An unsupervised clustering of prostate specimens based on contigs expression counts allowed proper discrimination of tumor from normal tissues of the Discovery Set (Fig. 2D).

In conclusion, DE-kupl identified thousands of PCa-associated RNA variants for the majority embedded into yet unreferenced transcripts which may represent putative novel lncRNAs. This depository was further explored for clinical relevance.

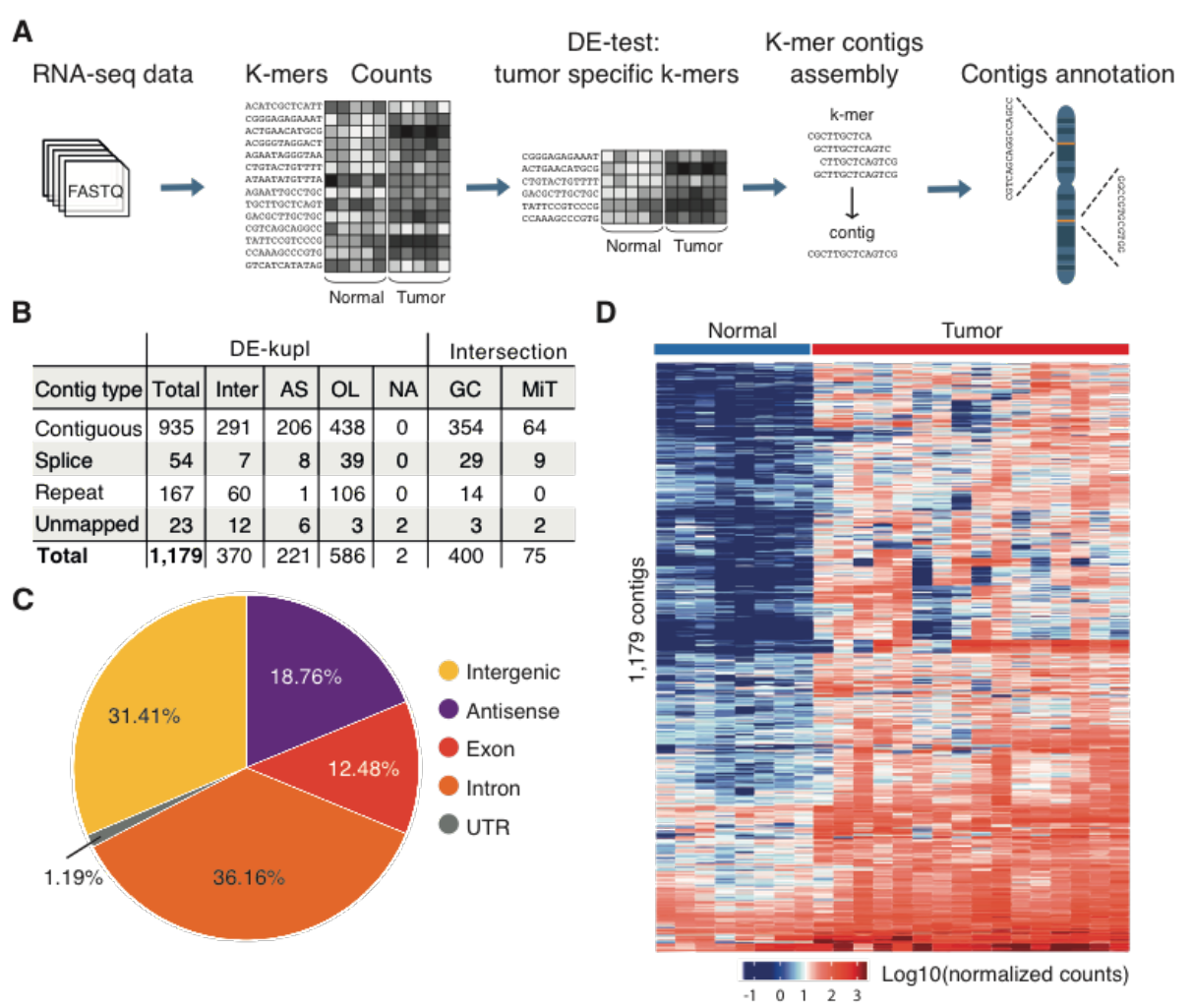

Figure 2. K-mer decomposition protocol for detection of differentially expressed RNA variants in PCa. (A) DE-kupl workflow with principle steps of contigs counting, DE-test and filtering, assembly and annotation. (B) Catalog of DE-kupl contigs of different subgroups: contiguous contigs mapped as unique fragments; spliced - contigs mapped as spliced fragments; repeat multiply mapped contigs; Inter - contigs mapping into intergenic regions, OL - overlapping GENCODE IncRNA annotations, AS - antisense to a protein-coding or a noncoding gene. Contigs of each subgroup showing 50\% sequence overlap with GENCODE (GC) and MiTranscriptome (MiT) annotated genes are counted. (C) Pie chart of contigs distribution across GENCODE annotated features. (D) Unsupervised hierarchical cluster heatmap of Log10(normalized counts) of 1,179 contigs assessed in 8 normal and 16 tumor specimens by total stranded RNA-seq of the Discovery Set. 


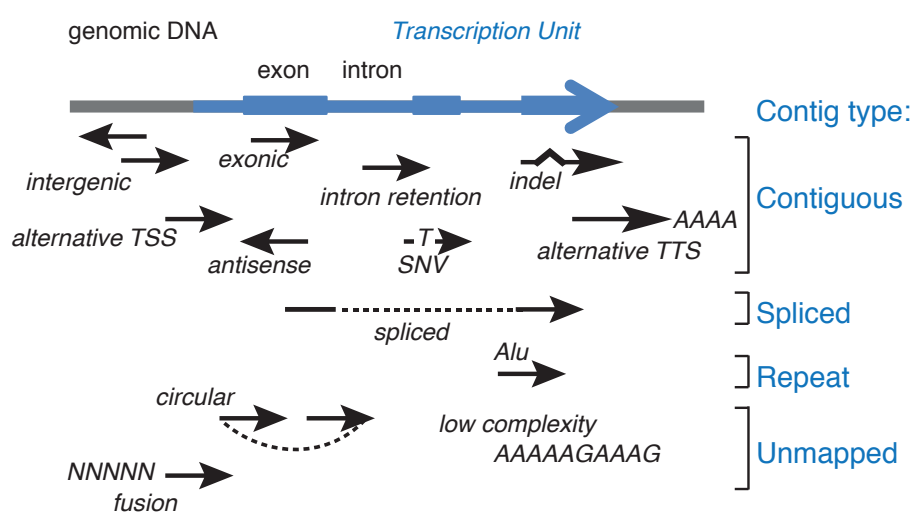

Figure S1. DE-kupl contigs assignment to contiguous, spliced, repeat and unmapped categories according to their genomic location outside or within annotated transcription units (blue). Each black arrow represents a contig.

\section{Naïve assembly of Transcription Units identifies novel prostate cancer associated IncRNAs}

To complement the reference-free protocol, we applied a reference-based protocol to build a catalog of IncRNAs from the same Discovery Set. Total RNA-seq produces much more intronic and exon-exon junction reads than poly(A)-selected RNA-seq, which is deleterious for splice graph-based assemblers such as Cufflinks (Kukurba and Montgomery 2015), (Hayer et al. 2015a). To bypass this difficulty, we developed a more straightforward IncRNA annotation pipeline, HoLdUp, which identifies transcription units (TU) based on coverage analysis (Fig. 3A). In this workflow, uniquely mapped reads were assembled into TUs and mapped to the GENCODE annotation to extract intergenic and antisense IncRNAs (see Methods for details). They were further ranked according to their expression level, presence of splice junctions and existence of matched expressed sequence tags (EST). In total, we retained 168,163 TUs with abovethreshold expression of 0.2 quartile of mRNA expression (Class 2) and, within this group, the most robust 2,972 TUs with at least one splice junction and one EST (Class 1) (Fig. 3B). Globally, newly detected transcripts were as much expressed as GENCODE annotated lncRNAs but lower than mRNAs (Fig. S2A). Only $0.33 \%$ of Class 1 lncRNAs 
bioRxiv preprint doi: https://doi.org/10.1101/644104; this version posted May 21, 2019. The copyright holder for this preprint (which was

not certified by peer review) is the author/funder, who has granted bioRxiv a license to display the preprint in perpetuity. It is made available under aCC-BY-NC-ND 4.0 International license.

were present with at least 50\% nucleotide sequence overlap in the recent GENCODE v26 catalog and $43.37 \%$ of TUs in the MiTranscriptome IncRNA repertoire; the rest represented putative novel lncRNA genes (Fig. 3B, Fig. S2B). Of 2,972 TUs, DE analysis retrieved 127 of Class 1 TUs significantly up-regulated in tumor specimens (adjusted pvalue below 0.01, DESeq), including multiple intergenic transcripts and transcripts antisense to protein-coding genes, such as HDAC9, TPO, FBXL7.

A

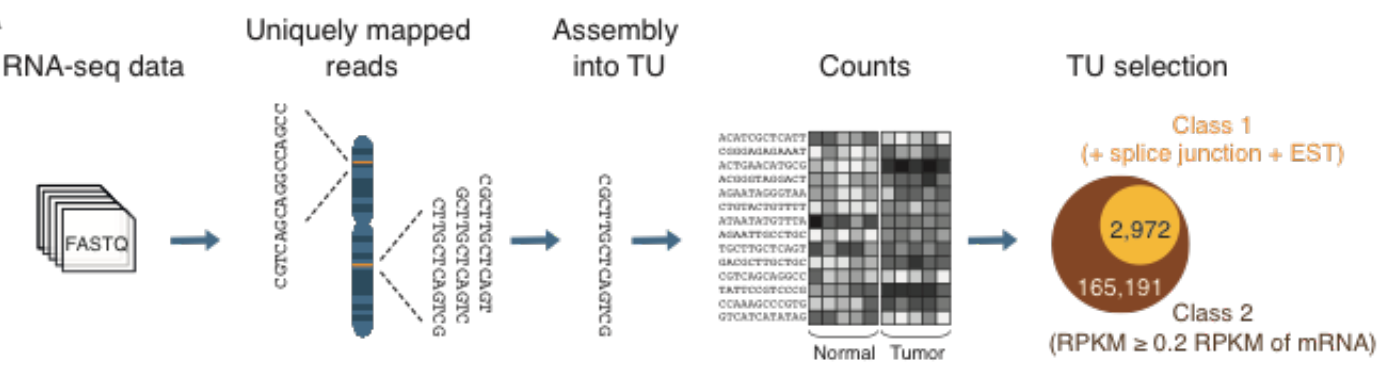

B

\begin{tabular}{lr|r|r}
\multicolumn{1}{l|}{ TU type } & & Class 2 & Class 1 \\
\hline & AS & 74,192 & 2,247 \\
\multirow{2}{*}{ Novel } & Inter & 93,971 & 725 \\
\cline { 2 - 4 } & $(\mathrm{GC})$ & $(336)$ & $(10)$ \\
& $(\mathrm{MiT})$ & $(10,753)$ & $(1,289)$ \\
\hline TOTAL & & 168,163 & $\mathbf{2 , 9 7 2}$ \\
(DE) & & $(2,487)$ & $(227)$
\end{tabular}

C

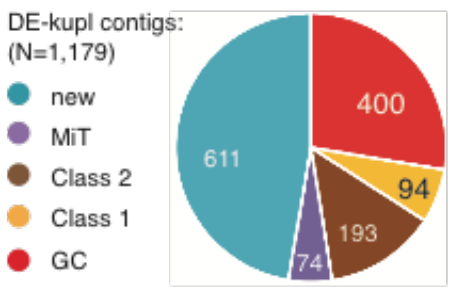

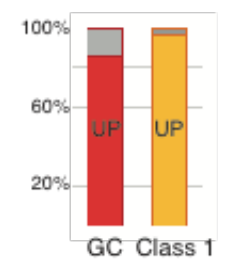

D

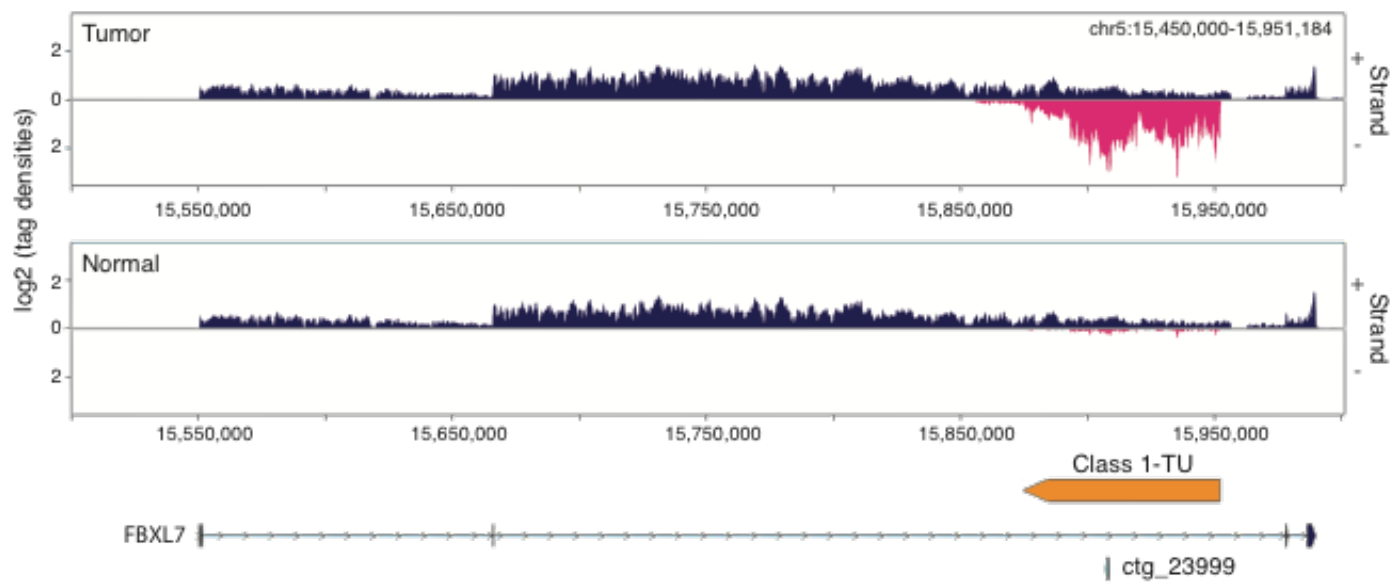

Figure 3. Reference-based IncRNA discovery from total stranded RNA-seq. (A) The HoLdUp protocol for the $a b$ initio assembly of TUs constituting putative IncRNA genes and their classification into Class 2 and Class 1 TUs according to robustness of detection. (B) HoLdUp catalog and TUs overlap with GENCODE v26 (GC) and MiTranscriptome (MiT) annotated IncRNAs. DE stands for differentially expressed transcripts (DESeq adj. p-value $<0.01$ ). (C) Pie chart representation of non-exclusive distribution of DE-kupl contigs across different IncRNA annotations: MiTranscriptome (violet), Class 1 (yellow), Class 2 (brown), GENCODE (red) and novel (blue), number of contigs is marked in each section. Proportion of DE-kupl contigs 
embedded into up-regulated (UP) GENCODE (red bar) and Class 1 (yellow bar) IncRNAs is expressed as a histogram. (D) Ving-generated RNA-seq profiling along plus (+) and minus (-) strands of chr5:15,500,295-15,939,910 in tumor and normal prostate specimens: the GENCODE annotated protein-coding gene FBXL7 (blue), antisense DE-kupl contig ctg_23999 (P22) and antisense HoLdUp Class 1-TU (orange). Arrow-lines and rectangles represent introns and exons, respectively. TU = transcription unit; $\mathrm{DE}=$ differentially expressed; RPKM = Reads per kilo base per million mapped reads.

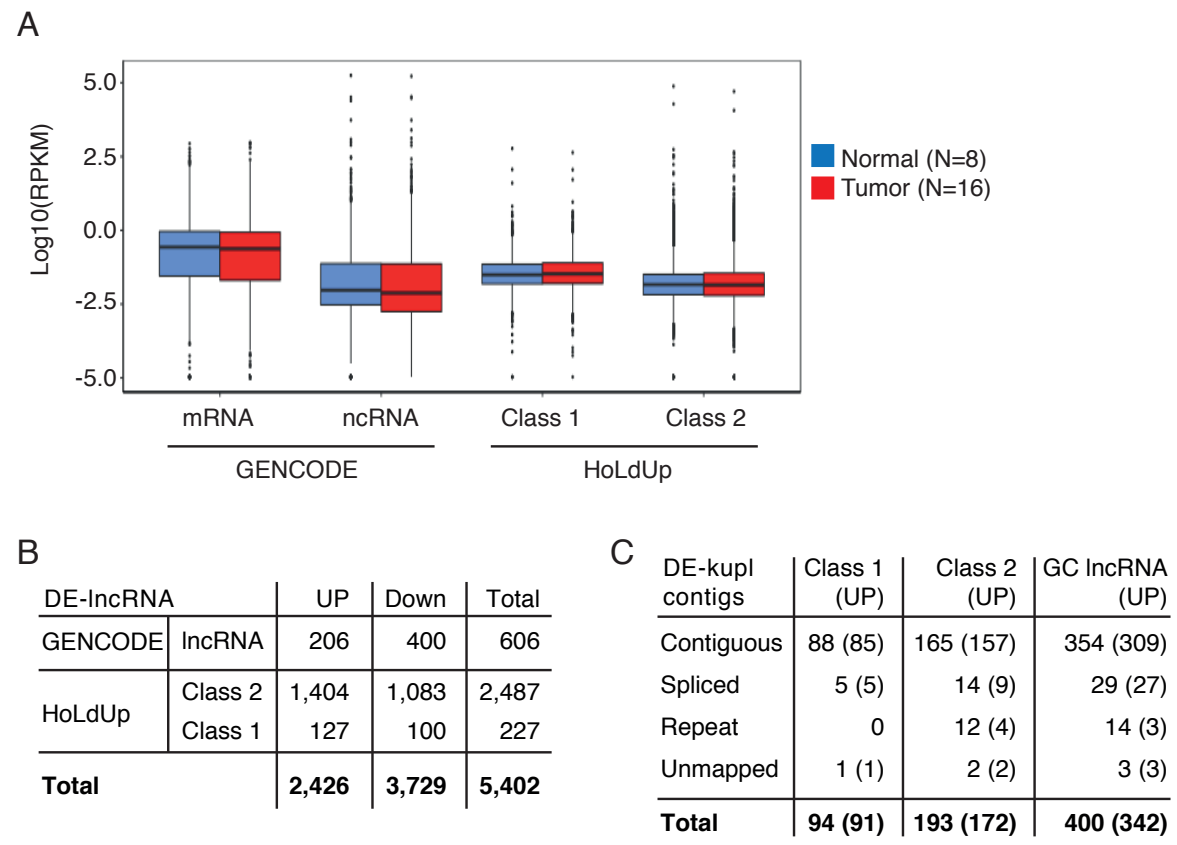

Figure S2. Expression of GENCODE and HoLdUp annotated lncRNAs in the Discovery Set. (A) Boxplot of Log10(RPKM) of mRNAs $(N=21,330)$, IncRNAs $(N=14,533)$ from the GENCODE annotation, and Class $1(\mathrm{~N}=2,967)$ and Class $2(\mathrm{~N}=168,163)$ TUs assembled by HoLdUp. Expression is measured in RPKM (Reads per kilo base per million mapped reads) by total stranded RNA-seq across 8 normal (bleu) and 16 tumor specimens (red). (B) Catalog of DE IncRNAs identified by DESeq within GENCODE IncRNAs and HoLdUp annotated TUs. (C) Intersection of DE-kupl contigs with HoLdUp and GENCODE annotated IncRNAs including those embedded into up-regulated transcripts (UP).

Intersection of DE-kupl contigs with HoLdUp TUs and the recent GENCODE IncRNA annotation showed that $687 \mathrm{DE}-\mathrm{kupl}$ contigs out of 1,179 make part of the stand-alone transcripts. Moreover, up to $85.5 \%$ and $96.8 \%$ DE-kupl contigs embedded into GENCODE and HoLdUp Class 1 lncRNA genes, respectively, were also detected by DESeq as significantly up-regulated transcripts in the same dataset, when the RNA-seq reads were counted within the entire TU (Fig. 3C; Fig. S2C). One such example is the contig 
ctg_23999 (P22) embedded into a novel HoLdUp assembled Class 1 TU antisense to the protein-coding FBXL7 gene (Fig. 3D).

In conclusion, the reference-based assembly protocol HoLdUp is complementary to DEkupl and allows attributing short RNA subsequences to whole transcription units. Nevertheless, DE-kupl was more powerful illuminating much more transcriptomic variations not only within the annotated loci but also within putative new noncoding regions in highly complex and heterogeneous total RNA-seq datasets of clinical origin.

\section{Selection of a restricted set of 23 PCa RNA contigs showing the highest differential} expression

We further leveraged the DE-kupl contig catalog to define a robust PCa signature among putative new IncRNAs using several filters (Fig. S3A). First, contigs were sorted according to their adjusted p-value and, second, were visually selected using the Integrative Genomic Viewer (IGV) applying the following criteria: (i) when several contigs were present within the same genomic region (5 kilobase window) the contig with the lowest adjusted p-value was retained, (ii) contigs antisense to expressed exons, bidirectional or positioned in close vicinity to other transcribed protein-coding genes were filtered out. We also retained contigs assigned to already known PCa associated IncRNAs, such as CTBP1-AS (ctg_25348, P10), PCAT7 (ctg_111158, P6) and PCAT1 (ctg_105149, P18), or lncRNAs referenced elsewhere as ctg_104447 (P11) mapped into LOC283177, ctg_123090 (P5) into AC004066.3, and ctg_73782 (P8) into LINC01006; all of which passed the aforementioned selection criteria. Notably, the RNA-seq visualization of a new contig antisense to the protein-coding FBP2 gene (ctg_28650, P2) revealed that it most likely makes part of the PCAT7 lncRNA as an extension of its last exon (Fig. S3B). The contig ctg_28650 (P2) was retained in the restricted list as the strongest candidate antisense to FBP2, overcoming ctg_111158 (P6) assigned to the PCAT7 gene. In total, 23 candidates belonging to contiguous $(\mathrm{N}=21)$, spliced $(\mathrm{N}=1)$ or repeat $(\mathrm{N}=1)$ subgroups of contigs were selected for further validation, all being 
expressed at least 6 times more in tumor tissues comparing to normal prostate (Fig. S3C, Supplemental Table S2). Among them, 12 candidates mapped antisense to annotated protein-coding or lncRNA genes and 11 located to intergenic regions. To facilitate further reading, contigs' identity (ID) are replaced by probes' ID from P1 to P23 according to increasing p-values of DE of the Discovery set (Supplemental Table S2).

A

\section{Contigs catalog $(\mathrm{N}=1,179)$ \\ 2. Best hits $\left(F C \geq 6, p\right.$-value $\left.\leq 10^{-4}\right)$ \\ 3. Contig visualization (IGV) \\ 4. One contig per gene $(\mathrm{N}=23)$ \\ 5. Expression measurements \\ PAIR $(\mathrm{N}=144) \quad$ TCGA-PRAD $(\mathrm{N}=557)$ \\ NanoString RNA-seq (polyA+, unstranded) \\ 6. Predictive modeling and $\mathrm{ROC}$ analysis \\ Multiplex Signature}

C

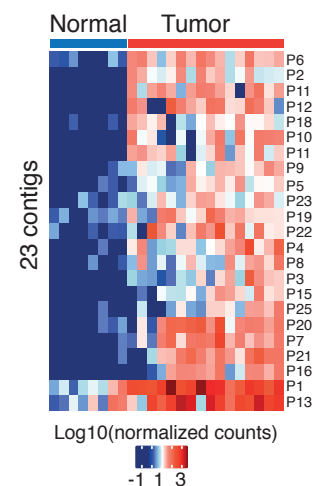

B

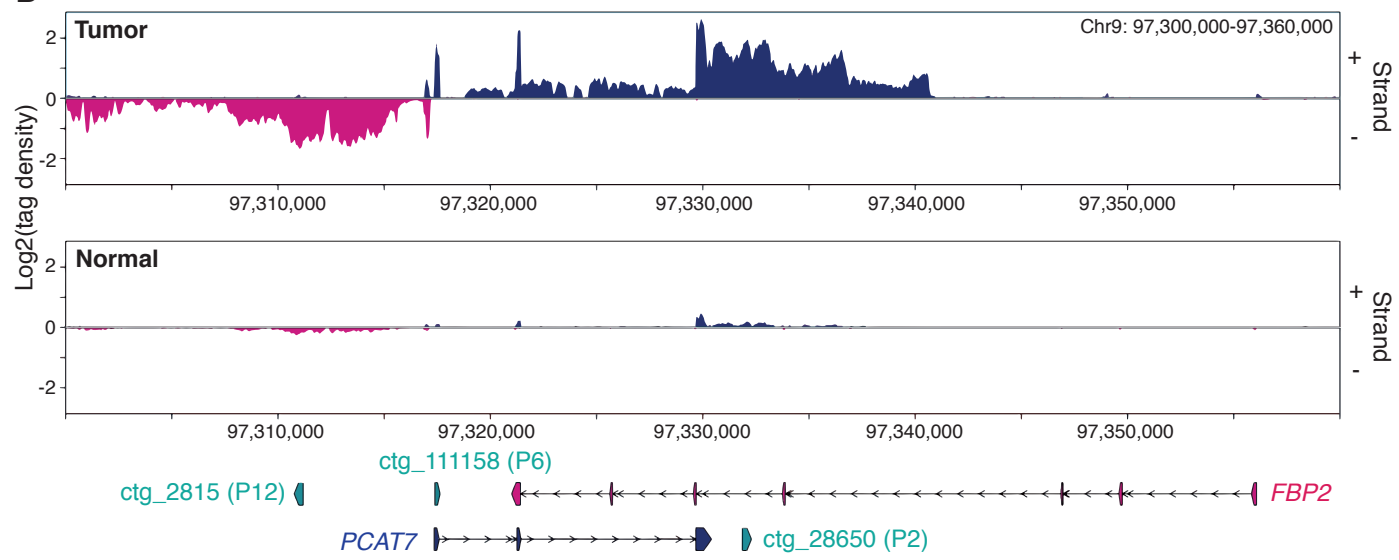

Figure S3. Selection of the most potent DE-kupl contigs. (A) Experimental rationale for contigs selection and validation of diagnostic potency. (B) RNA-seq profiling by VING along plus (+) and minus (-) strands of chr9: 97,300,000-97,360,000 in tumor and normal prostate specimens: the DE-kupl contig P6 (ctg_111158) assigned to PCAT7 and P2 (ctg_28650) antisense to the FBP2 gene. Arrow-lines represent introns, rectangles - exons. (C) Heatmap representing the expression level and unsupervised clustering of the selected 23 DE-kupl contigs across prostate cancer specimens of the Discovery Set.

Following the manual filtering we aimed to validate the expression of selected 23 contigs in the extended PAIR cohort of 9 normal and 135 tumor specimens (Selection 
Set) (Supplemental Table S3). For this purpose, an alternative RNA quantification procedure based on the NanoString nCounterTM platform for direct enzyme-free multiplex digital RNA measurements was carried out (Fig. 4A). In addition to DE-kupl contigs, a probe for PCA3 was used as a benchmark IncRNA. We also measured the expression of six housekeeping genes and selected three lowly expressed mRNAs (GPATCH3, ZNF2, ZNF346) as custom internal controls for relative quantifications (Supplemental Table S4, Fig. S4).

The NanoString assay revealed that all DE-kupl contigs were expressed at a lower level than PCA3, but still 21 out of 23 contigs were significantly overexpressed (Wilcoxon pvalue $<0.01$ ) in tumor specimens (Fig. 4A, Supplemental Table S5). Two contigs, intergenic P22 (ctg_119680) and repeat P17 (ctg_36195) did not show significant difference in expression between normal and tumor specimens. Ranking according to pvalues revealed 12 contigs better than PCA3. Among the top DE contigs were those embedded into PCAT1 (ctg_105149, P18), CTBP1-AS (ctg_25348, P10) and PCAT7 (ctg_111158, P6) genes, and the rest were assigned to novel lncRNAs. Notably, apart from P17 (ctg_36195) and P22 (ctg_119680), expression measurements were consistent between the two technologies, total stranded RNA-seq and NanoString, though the pvalues ordering was different (Fig. S5, Supplemental Table S6).

Thus, 21 out of 23 contigs were validated in the extended set of RNA specimens using the independent single-molecule measurement technology.

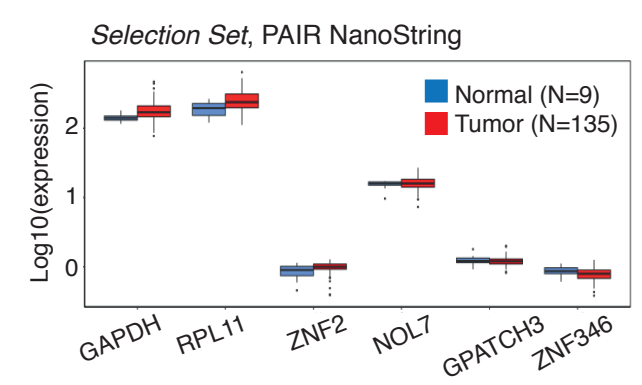

Figure S4. Box-plot of Log10(counts) of housekeeping protein-coding genes in 9 normal and 135 tumor specimens of the PAIR cohort (Selection Set) by the NanoString nCounter assay. 


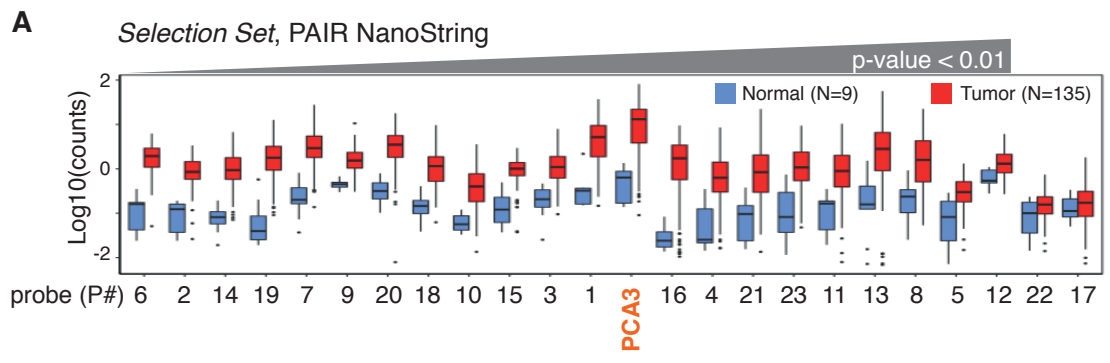

B Validation Set, TCGA-PRAD Poly(A)+ RNA-seq

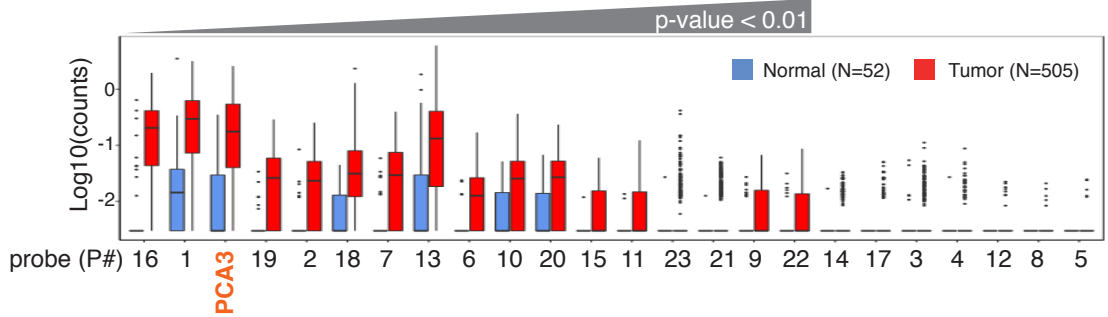

Figure 4. Expression of DE-kupl contigs in PAIR and TCGA-PRAD cohorts. (A) Box-plot of Log10(counts) of PCA3 and 23 DE-kupl contigs in 144 PAIR specimens of the Selection set by NanoString. (B) Box-plot of Log10(counts) of PCA3 and 23 DE-kupl contigs in 557 TCGA-PRAD specimens of the Validation Set by poly(A)+ unstranded RNA-seq. Normal tissues - in blue, tumor tissues - in red.

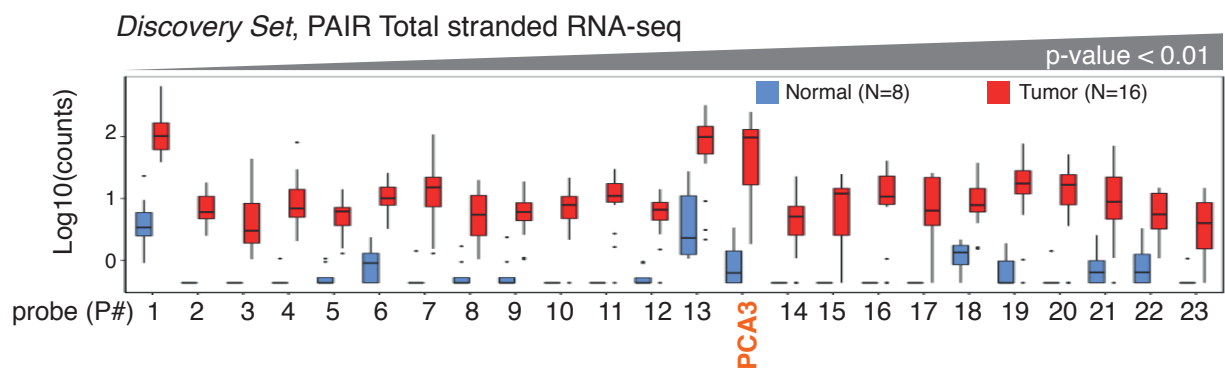

Figure S5. Box plot of PCA3 and DE-kupl contigs expression in the Discovery Set measured by the total stranded RNA-seq. Contigs are ordered by increasing adjusted p-values.

\section{Validation of contig-based RNA candidates in an independent clinical cohort}

Independent validation of DE-kupl contigs was done using the biggest PCa clinical resource of 557 poly(A)+ RNA-seq datasets, including 52 normal and 505 tumor tissues from radical prostatectomy (TCGA-PRAD cohort, Validation Set) (Fig. 1, Supplemental Table S7).

The occurrence of sequences representing 23 DE-kupl contigs was measured and compared to PCA3. In total, 16 out of 23 DE-kupl contigs had significant support for overexpression in tumor specimens in the TCGA-PRAD cohort (Wilcoxon p-value $<0.01$, 
FC > 2) (Fig. 4B, Supplemental Table S8). Among the best scored candidates, the two novel DE-kupl contigs, P16 (ctg_111348) antisense to DLX1 and intergenic P1 (ctg_17297), surpassed PCA3 ranked third. However, important discrepancies were observed between expression counts in poly(A)+ RNA-seq TCGA datasets and NanoString or total RNA-seq PAIR datasets. First, P22 (ctg_119680) was detected as DE in TCGA-PRAD, but failed the DE test when measured by NanoString (Fig. 4, Fig. S5). Second, the expression of nine DE-kupl contigs were near the base line in the TCGA dataset, including those showing relatively high expression and low p-values in the PAIR cohort, such as P14 (ctg_61528) antisense to TPO or the intergenic P9 (ctg_9446). Detection of these contigs in TCGA-PRAD was compromised independently of their genomic location (intergenic or antisense) or of the expression level of a sense-paired gene. We hypothesized that it is most likely due to a relatively low RNA-seq coverage and/or to a loss of poorly or non-polyadenylated transcripts during cDNA library preparation in the TCGA experimental setup. Finally, ranking of contigs according to increasing p-values was very different between the Validation, Discovery and Selection Sets highlighting remarkable discrepancies either between technologies or clinical origins.

Regardless all experimental biases, 16 out of 23 DE-kupl contigs were validated in the independent clinical cohort as significantly overexpressed in tumors. This cohort was further used for validation of clinical potency of contigs.

Expression of DE-kupl contigs is independent on tumor risk and recurrence metrics

Several clinical studies have revealed high heterogeneity of expression and low efficiency of the PCA3 biomarker in detection of high-risk tumors, questioning its robustness and reliability in PCa diagnostics (Alshalalfa et al. 2017), (Fenstermaker et al. 2017). We assessed contig expression in tumors of different clinical metrics. For risk prognosis, the most common metric is a three-group risk stratification system 
established by D'Amico in 1998 (D'Amico et al. 1998), which takes into account preoperative PSA level, biopsy Gleason score and clinical TNM stage. As mentioned above, this scheme is highly debated due to disagreements over the PSA score in relation to PCa over-diagnosis (Loeb et al. 2014), (Carlsson et al. 2012). To define a molecular signature independent of PSA, we excluded this criterion and categorized tumor specimens into low-, intermediate- and high-risk groups uniquely on the basis of Gleason and TNM features, below referred to as naïve indexing (Fig. S6). In addition to risk assessment, we also separated specimens in two subgroups depending on the tumor recurrence status (Fig. S6B). Then, expression of PCA3 and the 23 DE-kupl contigs were compared for each subgroup of the Selection Set.

\section{A}

\begin{tabular}{l|l|l} 
Risk group & Naïve (Gleason, TNM) & d'Amico (PSA, Gleason, TNM) \\
\hline Low-risk (LR) & Gleason 6/7 (3+4) and pT2 & $\begin{array}{l}\text { T1 - T2a and PSA }<10 \mathrm{ng} / \mathrm{ml} \\
\text { and Gleason } \leq 6\end{array}$ \\
\hline Intermediate-risk (IR) & $\begin{array}{l}\text { Gleason } 7(4+3) \text { and } \text { pT2, } \\
\text { Gleason }<8 \text { and pT3 }\end{array}$ & $\begin{array}{l}\text { T2b or PSA in between } 10-20 \mathrm{ng} / \mathrm{ml} \\
\text { or Gleason }=7\end{array}$ \\
\hline High-risk (HR) & Gleason 8/9 or pT3b/4 & $\begin{array}{l}\text { T2c }- \text { T3 or PSA }>20 \mathrm{ng} / \mathrm{ml} \text { or } \\
\text { Gleason } \geq 8\end{array}$
\end{tabular}

B

\begin{tabular}{|c|c|c|c|c|c|c|c|c|c|c|}
\hline \multirow[b]{2}{*}{ Source } & \multirow[b]{2}{*}{ Type of data } & \multirow[b]{2}{*}{ Total } & \multirow{2}{*}{$\begin{array}{l}\text { Normal } \\
\text { tissue }\end{array}$} & \multirow{2}{*}{$\begin{array}{l}\text { Tumor } \\
\text { tissue }\end{array}$} & \multicolumn{4}{|c|}{ Risk classification } & \multicolumn{2}{|c|}{ Recurrence } \\
\hline & & & & & $\mathrm{HR}$ & IR & LR & NA & NO & YES \\
\hline PAIR & $\begin{array}{l}\text { total stranded RNA-seq } \\
\text { NanoString }\end{array}$ & $\begin{array}{r}24 \\
144\end{array}$ & $\begin{array}{l}8 \\
9\end{array}$ & $\begin{array}{r}16 \\
135\end{array}$ & $\begin{array}{r}9 \\
49\end{array}$ & $\begin{array}{r}4 \\
51\end{array}$ & $\begin{array}{r}3 \\
35\end{array}$ & $\begin{array}{l}0 \\
0\end{array}$ & $\begin{array}{l}10 \\
80\end{array}$ & $\begin{array}{r}6 \\
55\end{array}$ \\
\hline TCGA-PRAD & $\begin{array}{l}\text { poly(A)+ unstranded } \\
\text { RNA-seq }\end{array}$ & 557 & 52 & 505 & 240 & 128 & 132 & 3 & 369 & 108 \\
\hline
\end{tabular}

Figure S6. Risk classification of prostate tumors according to their clinico-pathological features. (A) Risk prognosis classification criteria according to D'Amico and the PSA independent naïve indexing. (B) Risk classification and recurrence status of prostate specimens from TCGA-PRAD and PAIR cohorts used in this study. PSA=prostate specific antigen; TNM=tumor, node, metastasis; HR=high-risk, IR=intermediate-risk, LR=low-risk tumors.

To evaluate the robustness of contig expression, we ranked probes by decreasing FC for high-risk (HR) against low-risk (LR) tumors and positive versus negative recurrence status (Fig. 5). 

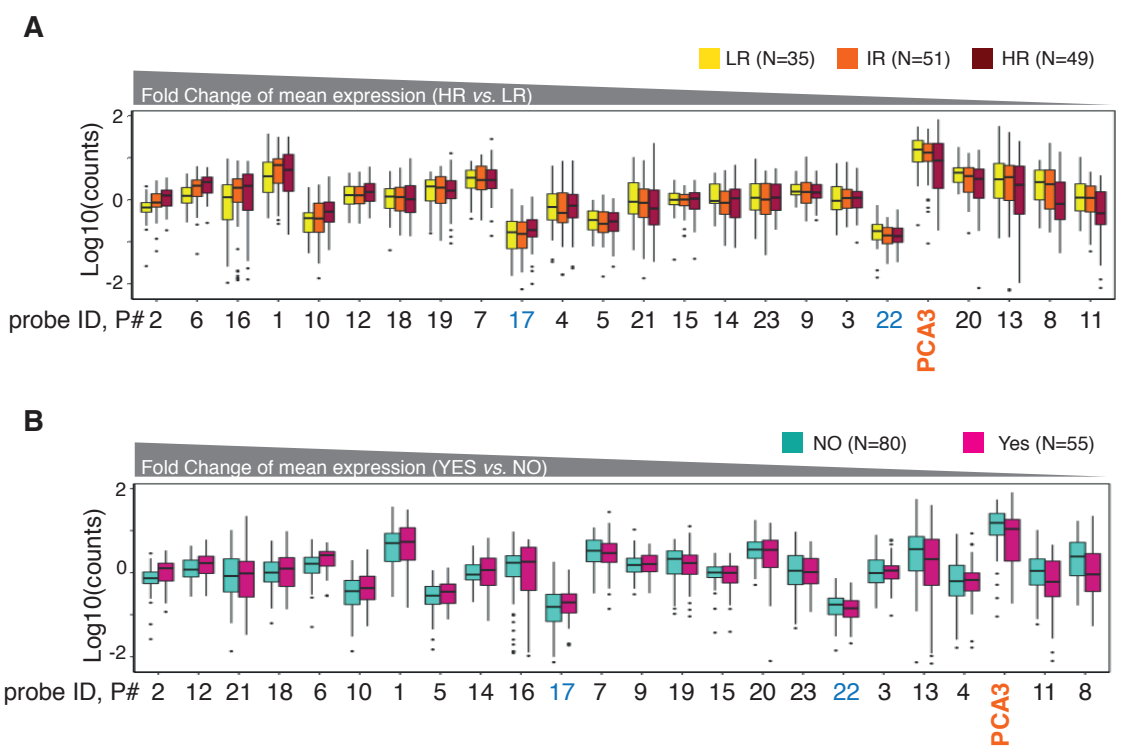

Figure 5. Box-plot of Log10(counts) of PCA3 and DE-kupl contigs in prostate specimens of the PAIR cohort (Selection Set) depending on tumor risk. (A) and recurrence status (B) assessed by NanoString. PCA3 is marked in orange, and the contigs showing insignificant expression change between normal and tumor specimens are in blue. Contigs are ordered by the decreasing FC of mean expression in HR vs. LR specimens in the A panel and in Yes vs. NO recurrence specimens in the B panel.

The majority of contigs showed robust expression independently of the tumor classification. In contrast, the PCA3 level was more disperse with the lower median and mean expression and higher p-values in high-risk and recurrence positive specimens (Supplemental Table S9). While considering only 21 significantly overexpressed contigs, 17 of them outperformed PCA3 in both contrasts (Supplemental Table S9). Notably, contigs P6 (ctg_111158) and P2 (ctg_28650) both antisense to FBP2, P10 (ctg_25348) embedded into CTBP1-AS, but also the novel P16 contig (ctg_111348) antisense to DLX1 and the intergenic P1 (ctg_17297) performed best.

In conclusion, the majority of DE-kupl contigs showed robust expression independent of tumor metrics. Hence, even if used alone, they may offer a better clinical potency for PCa diagnosis than PCA3.

Inferring a multiplex RNA-probe panel and evaluation of its performance in PCa

\section{diagnosis}


To extract parsimonious probe subsets predicting the tumor status, we applied LASSO (Least Absolute Shrinkage and Selection Operator) logistic regression on the Selection Set of 144 PAIR specimens (Ghosh and Chinnaiyan 2005). First, the initial 21 DE-kupl contigs and PCA3 validated for expression by NanoString were submitted to LASSO to define the best mixed signature comprised of already known and yet unannotated IncRNA probes for discrimination of tumor from normal tissues (Fig. S7A). Then, LASSO was performed with the probe subset composed uniquely of contigs assigned to putative novel lncRNAs $(\mathrm{N}=15)$ to infer the best new-lnc RNA signature. It resulted in two panels of 9 mixed and 9 new-lnc RNA candidates (Fig. 6A, Fig. S7B). Retrieved signatures were then used to predict a tumor status in the Validation Set of the TCGA-PRAD cohort using a leave-one-out cross-validated boosted logistic regression. To assess the sensitivity of DE-kupl contigs in PCa diagnosis, a predictive accuracy index, Area Under Curve (AUC) of the receiver-operating characteristic (ROC), was calculated for each signature and PCA3 alone in the PAIR (Selection Set) and TCGA-PRAD (Validation Set) datasets (Fig. 6B; Fig. S7B). Remarkably, all signatures still hold their predictive capacity in the independent TCGA-PRAD cohort in spite of the important differences in experimental setups between the two studies. Both markedly outperformed PCA3 for tumor detection with AUC of 0.92 for mixed and of 0.91 for new-lnc signatures against AUC of 0.73 for PCA3 (Fig. 6B and 6C). In addition, these signatures were much better in predicting high-risk tumors where PCA3 is particularly inaccurate (Fig. 6C). Remarkably, the newlnc RNA signature of 9 contigs composed uniquely of yet unannotated lncRNAs predicted the tumor status with the same performance as the mixed signature. Logistic regression did not retain PCA3 within the mixed signature set, instead contigs embedded into the well characterized PCAT1 IncRNA and into two already annotated but yet functionally uncharacterized IncRNAs LOC283177 and LINC01006 were present. 

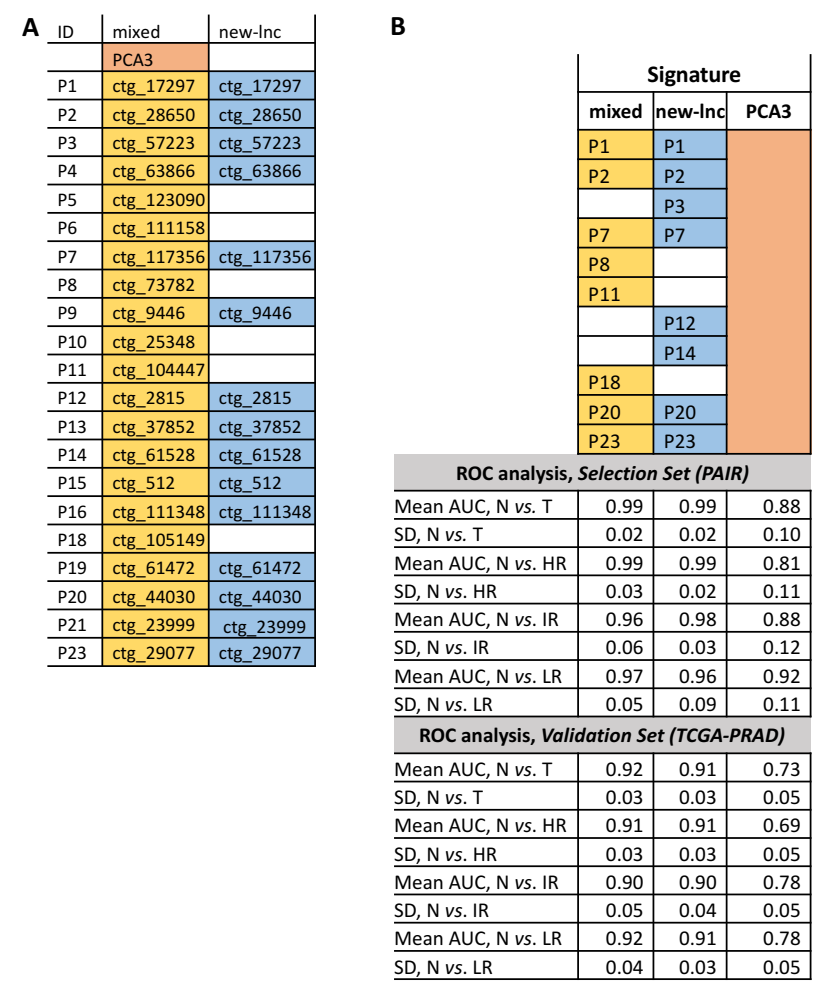

Figure S7. Predictive modeling and clinical performance analysis of DE-kupl contigs. (A) LASSO processed list of probes. (B) PCA3, mixed and new-lnc RNA signatures and their performance (mean and standard deviation of AUCs) in the PAIR (Selection Set) and the TCGA-PRAD (Validation Set) datasets. $\mathrm{N}=$ normal tissue, $\mathrm{T}=$ tumor tissue, $\mathrm{HR}=$ high-risk, $\mathrm{IR}=$ intermediaterisk, $\mathrm{LR}=$ low-risk tumors; $\mathrm{AUC}=$ area under the curve; $\mathrm{SD}=$ standard deviation.

\begin{tabular}{|c|c|c|c|c|c|}
\hline \multicolumn{6}{|c|}{ Signature } \\
\hline Probe & mixed & \multicolumn{2}{|c|}{ new-Inc } & \multicolumn{2}{|c|}{ contig origin } \\
\hline $\begin{array}{l}\text { P8 } \\
\text { P18 } \\
\text { P11 } \\
\text { P1 } \\
\text { P2 } \\
\text { P7 } \\
\text { P15 } \\
\text { P20 } \\
\text { P23 } \\
\text { P3 } \\
\text { P12 } \\
\text { P14 }\end{array}$ & \begin{tabular}{|l} 
ctg_73782 \\
ctg_105149 \\
ctg_104447 \\
ctg_17297 \\
ctg_28650 \\
ctg_117356 \\
ctg_512 \\
ctg_44030 \\
ctg_29077
\end{tabular} & \multicolumn{2}{|c|}{$\begin{array}{l}\text { ctg_17297 } \\
\text { ctg_28650 } \\
\text { ctg_117356 } \\
\text { ctg_512 } \\
\text { ctg_44030 } \\
\text { ctg_29077 } \\
\text { ctg_57223 } \\
\text { ctg_2815 } \\
\text { ctg_61528 }\end{array}$} & \multicolumn{2}{|c|}{$\begin{array}{l}\text { LINC01006 } \\
\text { PCAT1 } \\
\text { LOC283177 } \\
\text { intergenic } \\
\text { AS to } F B P 2 \\
\text { AS to snoU13 } \\
\text { AS to PXDN } \\
\text { integenic } \\
\text { AS to AC011523.2 } \\
\text { intergenic } \\
\text { intergenic } \\
\text { AS to TPO }\end{array}$} \\
\hline \multicolumn{2}{|l|}{ C } & PCA3 & $\mathrm{mi}$ & & new-Inc \\
\hline \multicolumn{2}{|c|}{ Normal vs. Tumor } & $0.73 \pm 0.05$ & \multicolumn{2}{|c|}{$0.92 \pm 0.03$} & $0.91 \pm 0.03$ \\
\hline \multicolumn{2}{|c|}{ Normal vs. HR } & $0.69 \pm 0.05$ & \multicolumn{2}{|c|}{$0.91 \pm 0.03$} & $0.91 \pm 0.03$ \\
\hline \multicolumn{2}{|c|}{ Normal vs. IR } & $0.78 \pm 0.05$ & \multicolumn{2}{|c|}{$0.90 \pm 0.05$} & $0.90 \pm 0.04$ \\
\hline \multicolumn{2}{|c|}{ Normal vs. LR } & $0.78 \pm 0.11$ & \multicolumn{2}{|c|}{$0.92 \pm 0.04$} & $0.91 \pm 0.03$ \\
\hline
\end{tabular}

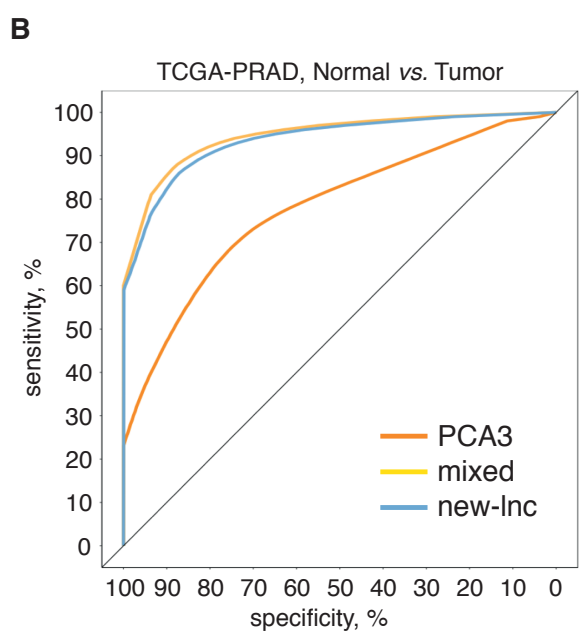

Figure 6. Predictive performance of PCA3 and multiplex mixed and new-lnc RNA signatures inferred from the LASSO penalized logistic regression. (A) Multiplex biomarker signatures composed of either known and unannotated RNAs (mixed) or of only unannotated RNAs (newlnc). (B) ROC for the PCa prediction in the TCGA dataset (Validation Set) using two signatures and PCA3 alone. (C) Mean and standard deviation of AUC computed over 100 samplings of the Validation Set for PCA3 and two signatures to classify tumors according to their risk status. AS = 
antisense; AUC = area under the curve; $\mathrm{HR}=$ high-risk, $\mathrm{IR}=$ intermediate-risk, $\mathrm{LR}=$ low-risk tumors.

This result highlights both the incompleteness of current cancer transcriptome datasets and the biological value of transcript information that can be extracted through adequate experimental (total stranded RNA-seq and NanoString quantification) and computational (DE-kupl) tools. The resulting signature demonstrated a sensitivity and robustness towards tumor risk surpassing the state of the art for discrimination of prostate cancer. Furthermore, the nine-probe RNA signature performed independently of tumor origin and clinico-pathological characteristics, but also independently of the technology used for RNA measurements.

\section{DISCUSSION}

Molecular biomarker assays are invaluable tools in cancer diagnosis, prognosis and treatment follow-up. Within this scope, sequencing technologies unveiled the pervasiveness and diversity of the human transcriptome, promoting lncRNAs as important cancer signatures (Schmitt and Chang 2016). These molecules are highly dynamic and reflect cellular states in a sensitive and specific way due to their involvement in genetic and regulatory flows of information. However, the variety of RNA forms and high heterogeneity of expression present a challenge for their detection and proper quantification in clinical samples. Predominant microarray and unstranded poly(A)+ RNA-seq based approaches allowed identification of numerous lncRNAs with tumorigenic function. However, their clinical performance as biomarkers stays rather poor due to the aforementioned RNA features hindering RNA detection, quantification and clinical validation under conventional experimental setups. Here, we presented an innovative experimental and computational platform that permits discovery of RNA biomarkers of high clinical potency from total stranded RNA-seq datasets of clinical origin. 
As a proof-of-concept, we focused on PCa as the only type of cancer using, so far, an RNA-based diagnostic test (ProgensaTM). The Discovery Set based on comparison of 8 normal with 16 tumor specimens from total RNA-seq datasets was processed by DEkupl to extract the most significant differentially expressed subsequences in the form of k-mer contigs. Further filtering based on contig length, genomic position and expression levels powered the pipeline towards the discovery of putative lncRNAs, for the majority, yet unreferenced in the human transcriptome. Then, the catalog of contigs was manually refined and tested for expression using the NanoString single-molecule RNA counting technology in the extended cohort of 144 specimens. Contig expression was next assessed in the independent, publicly available TCGA-PRAD dataset generated by the poly(A)+ unstranded RNA-seq technology. The expression of contigs was systematically compared to that of the benchmark biomarker IncRNA, PCA3. In total, 16 out of 23 contigs were validated in both setups but with important differences. Primarily, RNA measurements were consistent between two different technologies: NanoString and total stranded RNA-seq. In contrast, the TCGA poly(A)+ unstranded datasets revealed weakness and high heterogeneity of contig counts over the selected regions, resulting in unexpectedly low signals even for PCA3, considered as a highly expressed lncRNA. Our results promote the total stranded RNA-seq as a first-choice strategy for discovery of RNA biomarkers from clinical samples and when searching for transcripts others than highly abundant mRNAs. It reflects far more precisely the transcriptomic landscape of clinical samples and, hence, is more advantageous as a Discovery Set for development of clinical tests. At the same time, full-length transcript assembly from short-read sequencing is inaccurate, time and computer memory consuming, and this is aggravated by the added complexity of total (ribo-depleted) RNA-seq libraries (Hayer et al. 2015b). DE-kupl bypasses this issue by directly extracting from raw data RNA subsequences significantly overexpressed in a defined condition. In PCa tissues, this allowed identification of 1,179 lncRNA-hosted candidates. Further analysis isolated a restrained 
set of 9 contigs either within putative new IncRNAs or mixed annotated and novel IncRNAs allowing PCa diagnosis independently of tumor risk classifications with higher than the actual PCA3. Remarkably, the best performing mixed signature did not include PCA3, consistent with the low potency of this biomarker in detection of aggressive tumors. Instead, both mixed and new-lnc RNA signatures contained contigs embedded into putative novel lncRNA genes whose function in PCa progression will be important to explore. Among them, the P23 contig (ctg_29077) is antisense to AC011523.2, an intergenic lncRNA, co-transcribed with P23 in PCa specimens. This region is part of a super-enhancer, annotated in several PCa cell lines, located between KLK15 and the PSA encoding KLK3 genes (Jiang et al. 2019). Moreover, it has also been described as an enhancer bi-directionally transcribed into enhancer (e)RNAs and regulating expression of the neighboring $K L K 3$ and $K L K 2$ genes through eRNA and Med1-dependent chromatin looping in androgen-dependent LNCaP and VCaP, and androgen-independent LNCaP-abl cells (Hsieh et al. 2014). Presence of the P23 contig within the mixed and new-lnc RNA signatures supports, in addition to clinical potency, a possible regulatory function of the k-mer containing RNA contigs inferred by DE-kupl. More globally, the majority of DEkupl contigs within co-transcribed sense-antisense pairs were annotated as superenhancers in prostate tissues and cell lines or other biosamples, e.g. P15 (ctg_512), P7 (ctg_117356), and P4 (ctg_63866) (Jiang et al. 2019). In most cases, their function in gene expression regulation and chromatin configuration has not yet been investigated and experimentally validated, but it is tempting to speculate that defined senseantisense transcripts may influence a super-enhancer activity and, consequently, may fine-tune the expression of neighboring genes.

In this work, we propose DE-kupl as a tool for discovery of novel disease-associated transcriptomic variations, which can be further explored for biological and clinical relevance. As a pilot project, we oriented the pipeline towards the discovery of novel IncRNAs, but using proper masking and filtering criteria defined by the investigator, 
other variant transcripts including single nucleotide variations (SNV), novel splice events, gene fusion, circular RNAs or exogenous viral RNAs could be probed. The workflow can be applied to any RNA-seq datasets of any clinical origin to generate a probe panel that may be implemented as a multiplex platform for simultaneous detection of RNAs in clinical samples. Moreover, different experimental contrasts (normal vs. pathology, low- vs. high-risk grade, treatment resistant vs. sensitive, etc.) will define the biomarker usage in diagnosis, prognosis or other clinical applications, hence, providing clinicians and researchers with a simple and highly sensitive tool for genomic and personalized medicine. 


\section{METHODS}

\section{Tissue samples}

Tumor and normal biopsy specimens were retrospectively collected from prostate cancer patients who provided informed consent and were approved for distribution by the H. Mondor institutional board (PAIR cohort). Tumors classification in low-, intermediate- and high-risk prognosis was performed according to Gleason and TNM scores and regardless PSA values (Supplemental Table S1, S3).

\section{RNA extraction, quantification and cDNA library production}

Total RNA was extracted using the TRizol reagent (ThermoFisher), according to manufacturer's procedure, quantified and quality controlled using a 2100 Bioanalyzer (Agilent). RNA samples with RNA Integrity Number (RIN) above 6 were depleted for ribosomal RNA and converted into cDNA library using a TruSeq Stranded Total Library Preparation kit (Illumina). cDNA libraries were normalized using an Illumina Duplexspecific Nuclease (DSN) protocol prior to a paired-end sequencing on $\mathrm{HiSeq}^{\text {TM }} 2500$ (Illumina). At least 20x coverage per sample was considered as minimum of unique sequences for further data analysis.

\section{RNA-sequencing data}

Raw paired-end strand-specific RNA-seq data was generated by our laboratory from ribo-depleted total RNA samples of prostate tissues (8 normal and 16 tumor specimens; Supplemental Table S1) and can be retrieved from the gene omnibus portal (GEO), accession number GSE115414.

TCGA prostate cancer poly(A)-selected RNA-seq and corresponding clinical data were obtained from publicly available TCGA dataset (http://cancergenome.nih.gov), 557 inputs in total (52 normal and 505 tumors of high- $(\mathrm{N}=240)$, intermediate- $(\mathrm{N}=128)$ and low-risk (N=132) groups. Among them, 369 patients showed no tumor recurrence, 108 presented a new tumor event (Supplemental Table S7). 


\section{Computational workflow for k-mer contigs discovery from total stranded RNA-seq dataset}

DE-kupl run was performed from (June 2017) with parameters ctg_length 31, min_recurrence 6, min_recurrence_abundance 5, pvalue_threshold 0.05 , lib_type stranded, diff_method DESeq2. K-mer masking was performed against the GENCODE v24 annotation. DE-kupl analysis of the 8 against 16 PAIR RNA-seq prostate libraries yielded 124,809 DE contigs, in total. Contigs were annotated by alignment on the hg19 human genome assembly using the DE-kupl annotate procedure. We further selected contigs of size above 200 nucleotides and classified them into four categories (contiguous, repeat, spliced, unmapped) based on their location and mapping features.

\section{Computational workflow for reference-based $a b$ initio transcripts assembly from} total stranded RNA-seq dataset (HoLdUP)

The human genome version hg19 and the GENCODE v14 annotation were used in this study. First, we performed a quality control of all sequencing data by FastQC Babraham Bioinformatics software. Reads were mapped using TopHat 2.0.4, allowing 3 mismatches and requesting uniquely mapped reads which were further assembled using the BedTools suite. Overlapping contigs from all libraries were merged and only contigs supported by at least 10 reads in either library were further assembled in segments if mapped in the same strand and separated by less than 100 nucleotides. We compared segments to the GENCODE v14 annotation to extract antisense and intergenic TU longer than 200 nucleotides. To classify lncRNAs, we applied the following criteria: (i) an expression level above 0.2 quartile of mRNA expression in at least one condition per tissue (Class 2); (ii) within this class, all TUs containing at least one TopHatidentified exon-exon junction and at least one spliced EST from UCSC mapped contigs were assigned to Class 1 . The whole catalog, the R code and Data Tables can be provided upon request.

Overlap between GENCODE, MiTranscriptome, DE-kupl and HoLdUp catalogues 
Intersection between transcripts was counted only in case of $50 \%$ overlap of nucleotide sequence between genomic coordinates of each fragment.

\section{Differential expression analysis}

Read counting was performed on the compiled annotation (GENCODE v26, HoLdUp Class 1 and Class 2) for each sample, using featureCounts 1.6.0 with the following parameters: -F "SAF" -p -s 2 -O and the DESeq R package (Love et al. 2014). Only RNAs with adjusted p-value below 0.01 were retained as differentially expressed to constitute the prostate tumor signature.

\section{NanoString nCounter Expression Assay}

$100 \mathrm{ng}$ of total RNA was used for direct digital detection of 29 target transcripts: 6 housekeeping genes (RPL11, GAPDH, NOL7, GPATCH3, ZNF2 and ZNF346), 23 contigs and the one known PCa-associated lncRNA, PCA3. Each target gene of interest was detected in RNA samples of 144 specimens (9 normal and 135 tumor) of the PAIR cohort (Supplemental Table S3) on NanoString nCounter V2 using reporter and capture probes of 35- to 50-nucleotide targeting sequences. Data was normalized through the use of NanoString's intrinsic positive controls and then contig expression was calculated relative to the average signal of three housekeeping genes (GPATCH3, ZNF2 and ZNF346). Raw and normalized data for each specimen, mean and fold change expression in normal against tumor samples are presented in Supplemental Table S4 and S5.

\section{Contig expression measurements in TCGA-PRAD datasets}

DE-kupl provides representative k-mers for each differentially expressed contig. We converted the TCGA-PRAD FASTQ files to k-mer counts using Jellyfish count and counted representative k-mers in each Jellyfish count file using the Jellyfish query command. Counts were normalized by total number of reads in corresponding libraries. To determine whether counts of DE-kupl derived representative k-mer were a reliable proxy for evaluating contig expression, we compared representative k-mer counts to average counts from k-mers sampled along each contig. All individual counts were 
obtained using Jellyfish Dump files produced for each TCGA-PRAD library. Sampling was performed as follows: (i) we extracted all k-mers from the contig that were unique in the Ensembl human v91 transcript reference, and (ii) from this list we sampled 10 regularly spaced k-mers, starting from the first $10 \%$ and ending in the last $10 \%$ of the list. This sampling procedure was repeated four times for each contig. For the whole TCGA library and each contig, the $10 \mathrm{k}$-mer counts obtained by Jellyfish were averaged, yielding one average count per sample per library. Correlations between sample counts and representative k-mer counts are shown in Fig. S8 for two DE-kupl contigs.

A
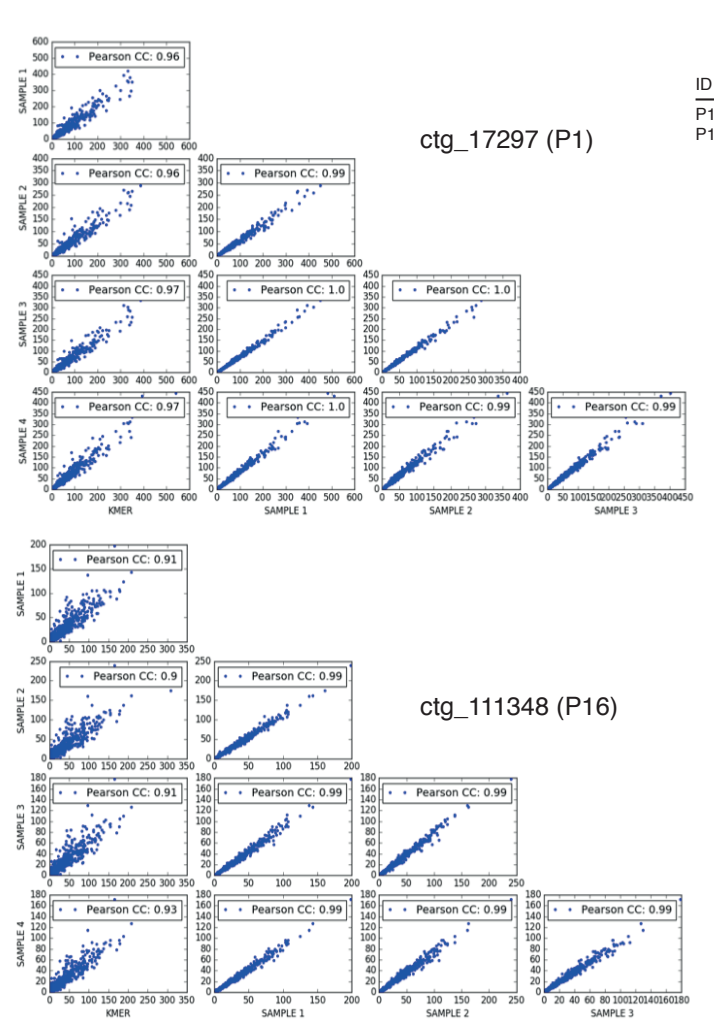

B

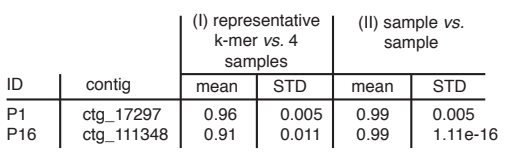

Figure S8. Contig expression measurements in TCGA-PRAD datasets. (A) Stability of k-mer counts for contigs P1 (ctg_17297) and P16 (ctg_111348) across the TCGA-PRAD dataset. (B) Pearson correlations between counts of representative k-mers and sampled k-mers from the same contig: for each contig, in the TCGA-PRAD datasets $(\mathrm{N}=557)$ the number of occurrences of (I) the representative DE-kupl k-mer and (II) of four sets of $10 \mathrm{k}$-mers sampled at regular distance along the length of the contig. Each sample (noted SAMPLE1-4) was obtained by changing the starting position of the first k-mer.

\section{RNA-sequencing data visualization}


RNA-seq reads profiling along a locus of interest was performed using our in-house $\mathrm{R}$ script VING (Descrimes et al. 2015). The normal samples were assigned to the group "controls" and the tumor specimens - to the group "cases", with the assumption that the "cases" should have higher values than "controls".

\section{Unsupervised clustering of prostate specimens}

Specimens were ranked based on the Log10(expression counts) levels of contigs assessed by the NanoString nCounter assay using a ComplexHeatmap R-package (Gu et al. 2016).

\section{Variable selection using the LASSO penalized logistic regression and external validation of signatures}

Signature inference was performed in R using the normalized Selection Set (23 probes in 144 observations) as a variable selection dataset and contigs counts table of the Validation Set (23 probes in 557 observations) as an external validation dataset (R Core Team). First, we performed penalized logistic regression using the glmnet R package to select probes predicting the tumor status on the Selection Set upsampled to correct the imbalance class distribution (9 normal versus 135 tumor specimens) (Friedman et al. 2010). Selection was performed using all probes (signature_mixed including PCA3) or using only new-lnc RNA contigs only (signature_new-lnc) (Fig. S7). Second, we built predictors using the boosted logistic regression from the caTools and caret packages (Tuszynski 2008), (Kuhn 2008). AUCs were computed using the precrec package on 100 training and testing datasets (Saito and Rehmsmeier 2017), sub-sampled from the initial dataset (Normal vs. Tumor, Normal vs. HR, Normal vs. IR and Normal vs. LR) using the sample.split function from the caTools package.

\section{DATA ACCESS}

TCGA prostate cancer poly(A)-selected RNA-seq and corresponding clinical data can be obtained from TCGA portal (https://www.cancer.gov/tcga).

\section{ACKNOWLEDGEMENTS}


We deeply thank Dominika Foretek and Maxime Wery for editorial suggestions, Camille Gautier, Claire Bertrand and Anna Almeida (Morillon lab, Institut Curie) and Sylvain Baulande for RNA-seq (NGS platform, Institut Curie), Audrey Rapinat and David Gentien for NanoString experiments (Genomic Platform, Institut Curie), Cedric Saule and Jeremy Le Coz for a DE-kupl run (Gautheret lab, I2BC). TCGA RNA-seq data were downloaded from the dbGaP website under authorization phs000178/GRU to D.G..

\section{DISCLOSURE DECLARATION}

The authors declare no competing interests.

\section{FUNDING}

Constitution of the cancer prostate cohort received financial support through a grant from the INCa-Ligue-ARC PAIR program (to Y.A. and A.L-V.). RNA-seq efforts were supported by a grant from the ICGex program at Institut Curie (to A.L-V., A.M.) and benefited from the facilities and expertise of the NGS platform of Institut Curie, supported by Agence Nationale de la Recherche (ANR-10-EQPX-03, ANR10-INBS-09-08) and Canceropôle Ile-de-France. M.D., Ma.G., A.M., M.P., and Z.S. were supported by Agence Nationale de la Recherche (DNA-Life) and the European Research Council (ERCconsolidator DARK-616180-ERC-2014) attributed to A.M.; D.G. and N.H.N. were supported by ITMO Cancer - Systems Biology (bio2014-04) and Agence Nationale de la Recherche "France Génomique" (ANR-10-INBS-0009) attributed to D.G..

\section{AUTHORS' CONTRIBUTIONS}

Conceptualization: D.G., A.M..

Funding acquisition: A.M., D.G..

Data acquisition: M.P..

Clinical samples collection and classification: Y.A., V.F., A.T..

Data analysis and curation: M.D., Mé.G., Ma.G., N.H.N., Z.S., M.P..

Interpretation: Y.A., D.G., V.F., A. L.-V., A.M., M.P., A.T..

Writing, review, and/or revision of the manuscript: D.G., A.M., M.P., Mé.G.. 
Study supervision: D.G., A.M., M.P..

\section{REFERENCES}

Alshalalfa M, Verhaegh GW, Gibb EA, Santiago-Jiménez M, Erho N, Jordan J, Yousefi K, Lam LLC, Kolisnik T, Chelissery J, et al. 2017. Low PCA3 expression is a marker of poor differentiation in localized prostate tumors: exploratory analysis from 12,076 patients. Oncotarget $\mathbf{8}$.

Audoux J, Philippe N, Chikhi R, Salson M, Gallopin M, Gabriel M, Le Coz J, Drouineau E, Commes T, Gautheret D. 2017. DE-kupl: exhaustive capture of biological variation in RNA-seq data through kmer decomposition. Genome Biol $\mathbf{1 8 .}$

Carlsson S, Vickers AJ, Roobol M, Eastham J, Scardino P, Lilja H, Hugosson J. 2012. Prostate Cancer Screening: Facts, Statistics, and Interpretation in Response to the US Preventive Services Task Force Review. J Clin Oncol 30: 2581-2584.

D’Amico AV, Whittington R, Kaplan I, Beard C, Schultz D, Malkowicz SB, Wein A, Tomaszewski JE, Coleman CN. 1998. Calculated prostate carcinoma volume: The optimal predictor of 3-year prostate specific antigen (PSA) failure free survival after surgery or radiation therapy of patients with pretreatment PSA levels of 4-20 nanograms per milliliter. Cancer 82: 334-341.

Davila JI, Fadra NM, Wang X, McDonald AM, Nair AA, Crusan B R, Wu X, Blommel JH, Jen J, Rumilla KM, et al. 2016. Impact of RNA degradation on fusion detection by RNA-seq. BMC Genomics 17. http://bmcgenomics.biomedcentral.com/articles/10.1186/s12864-016-3161-9 (Accessed May 7, 2018).

de Kok JB, Verhaegh GW, Roelofs RW, Hessels D, Kiemeney LA, Aalders TW, Swinkels DW, Schalken JA. 2002. DD3(PCA3), a very sensitive and specific marker to detect prostate tumors. Cancer Res 62: 2695-2698.

Deng J, Tang J, Wang G, Zhu Y-S. 2017. Long Non-Coding RNA as Potential Biomarker for Prostate Cancer: Is It Making a Difference? Int J Environ Res Public Health 14.

Descrimes M, Ben Zouari Y, Wery M, Legendre R, Gautheret D, Morillon A. 2015. VING: a software for visualization of deep sequencing signals. BMC Res Notes 8: 419.

Deveson IW, Brunck ME, Blackburn J, Tseng E, Hon T, Clark TA, Clark MB, Crawford J, Dinger ME, Nielsen LK, et al. 2018. Universal Alternative Splicing of Noncoding Exons. Cell Syst 6: 245-255.e5. Fenstermaker M, Mendhiratta N, Bjurlin MA, Meng X, Rosenkrantz AB, Huang R, Deng F-M, Zhou M, Huang WC, Lepor H, et al. 2017. Risk Stratification by Urinary Prostate Cancer Gene 3 Testing 
Before Magnetic Resonance Imaging-Ultrasound Fusion-targeted Prostate Biopsy Among Men With No History of Biopsy. Urology 99: 174-179.

Friedman J, Hastie T, Tibshirani R. 2010. Regularization Paths for Generalized Linear Models via Coordinate Descent. J Stat Softw 33: 1-22.

Galasso F, Giannella R, Bruni P, Giulivo R, Barbini VR, Disanto V, Leonardi R, Pansadoro V, Sepe G. 2010. PCA3: a new tool to diagnose prostate cancer (PCa) and a guidance in biopsy decisions. Preliminary report of the UrOP study. Arch Ital Urol Androl Organo Uff Soc Ital Ecogr Urol E Nefrol 82: $5-9$.

Ghosh D, Chinnaiyan AM. 2005. Classification and selection of biomarkers in genomic data using LASSO. J Biomed Biotechnol 2005: 147-154.

Groskopf J, Aubin SMJ, Deras IL, Blase A, Bodrug S, Clark C, Brentano S, Mathis J, Pham J, Meyer T, et al. 2006. APTIMA PCA3 molecular urine test: development of a method to aid in the diagnosis of prostate cancer. Clin Chem 52: 1089-1095.

Gu Z, Eils R, Schlesner M. 2016. Complex heatmaps reveal patterns and correlations in multidimensional genomic data. Bioinformatics 32: 2847-2849.

Hayer KE, Pizarro A, Lahens NF, Hogenesch JB, Grant GR. 2015a. Benchmark analysis of algorithms for determining and quantifying full-length mRNA splice forms from RNA-seq data. Bioinforma Oxf Engl 31: 3938-3945.

Hayer KE, Pizarro A, Lahens NF, Hogenesch JB, Grant GR. 2015b. Benchmark analysis of algorithms for determining and quantifying full-length mRNA splice forms from RNA-seq data. Bioinformatics btv488.

Hsieh C-L, Fei T, Chen Y, Li T, Gao Y, Wang X, Sun T, Sweeney CJ, Lee G-SM, Chen S, et al. 2014. Enhancer RNAs participate in androgen receptor-driven looping that selectively enhances gene activation. Proc Natl Acad Sci 111: 7319-7324.

Iyer MK, Niknafs YS, Malik R, Singhal U, Sahu A, Hosono Y, Barrette TR, Prensner JR, Evans JR, Zhao S, et al. 2015. The landscape of long noncoding RNAs in the human transcriptome. Nat Genet 47: 199-208.

Jarroux J, Morillon A, Pinskaya M. 2017. History, Discovery, and Classification of lncRNAs. Adv Exp Med Biol 1008: 1-46.

Jiang Y, Qian F, Bai X, Liu Y, Wang Q, Ai B, Han X, Shi S, Zhang J, Li X, et al. 2019. SEdb: a 
comprehensive human super-enhancer database. Nucleic Acids Res 47: D235-D243.

Kuhn M. 2008. Building Predictive Models in $R$ Using the caret Package. J Stat Softw 28. http://www.jstatsoft.org/v28/i05/ (Accessed April 3, 2019).

Kukurba KR, Montgomery SB. 2015. RNA Sequencing and Analysis. Cold Spring Harb Protoc 2015: $951-969$

Leucci E. 2018. Cancer development and therapy resistance: spotlights on the dark side of the genome. Pharmacol Ther 189: 22-30.

Loeb S, Bjurlin MA, Nicholson J, Tammela TL, Penson DF, Carter HB, Carroll P, Etzioni R. 2014. Overdiagnosis and Overtreatment of Prostate Cancer. Eur Urol 65: 1046-1055.

Loeb S, Partin AW. 2011. Review of the literature: PCA3 for prostate cancer risk assessment and prognostication. Rev Urol 13: e191-195.

Love MI, Huber W, Anders S. 2014. Moderated estimation of fold change and dispersion for RNA-seq data with DESeq2. Genome Biol 15: 550.

Morillon A, Gautheret D. 2019. Bridging the gap between reference and real transcriptomes. Genome Biology, in press

Prensner JR, Chen W, Iyer MK, Cao Q, Ma T, Han S, Sahu A, Malik R, Wilder-Romans K, Navone N, et al. 2014. PCAT-1, a long noncoding RNA, regulates BRCA2 and controls homologous recombination in cancer. Cancer Res 74: 1651-1660.

Prensner JR, Iyer MK, Sahu A, Asangani IA, Cao Q, Patel L, Vergara IA, Davicioni E, Erho N, Ghadessi M, et al. 2013. The long noncoding RNA SChLAP1 promotes aggressive prostate cancer and antagonizes the SWI/SNF complex. Nat Genet 45: 1392-1398.

Quinn JJ, Chang HY. 2015. Unique features of long non-coding RNA biogenesis and function. Nat Rev Genet 17: 47-62.

R Core Team. R: A language and environment for statistical computing. R Foundation for Statistical Computing. http://www.R-project.org/.

Saito T, Rehmsmeier M. 2017. Precrec: fast and accurate precision-recall and ROC curve calculations in R. Bioinformatics 33: 145-147.

Salameh A, Lee AK, Cardó-Vila M, Nunes DN, Efstathiou E, Staquicini FI, Dobroff AS, Marchiò S, Navone NM, Hosoya H, et al. 2015. PRUNE2 is a human prostate cancer suppressor regulated by the intronic long noncoding RNA PCA3. Proc Natl Acad Sci U S A 112: 8403-8408. 
Schmitt AM, Chang HY. 2016. Long Noncoding RNAs in Cancer Pathways. Cancer Cell 29: 452-463.

Silva A, Bullock M, Calin G. 2015. The Clinical Relevance of Long Non-Coding RNAs in Cancer. Cancers 7: 2169-2182.

Tuszynski J. 2008. caTools: Tools: moving window statistics, GIF, Base64, ROC AUC, etc. https://CRAN.R-project.org/package=caTools.

Uszczynska-Ratajczak B, Lagarde J, Frankish A, Guigó R, Johnson R. 2018. Towards a complete map of the human long non-coding RNA transcriptome. Nat Rev Genet 19: 535-548.

Van Grembergen O, Bizet M, de Bony EJ, Calonne E, Putmans P, Brohée S, Olsen C, Guo M, Bontempi G, Sotiriou C, et al. 2016. Portraying breast cancers with long noncoding RNAs. Sci Adv 2: e1600220.

Wang F, Ren S, Chen R, Lu J, Shi X, Zhu Y, Zhang W, Jing T, Zhang C, Shen J, et al. 2014. Development and prospective multicenter evaluation of the long noncoding RNA MALAT-1 as a diagnostic urinary biomarker for prostate cancer. Oncotarget 5.

Wang Y-H, Ji J, Wang B-C, Chen H, Yang Z-H, Wang K, Luo C-L, Zhang W-W, Wang F-B, Zhang X-L. 2018. Tumor-Derived Exosomal Long Noncoding RNAs as Promising Diagnostic Biomarkers for Prostate Cancer. Cell Physiol Biochem 46: 532-545.

Zhao S, Zhang Y, Gamini R, Zhang B, von Schack D. 2018a. Evaluation of two main RNA-seq approaches for gene quantification in clinical RNA sequencing: polyA+ selection versus rRNA depletion. Sci Rep 8: 4781.

Zhao S, Zhang Y, Gamini R, Zhang B, von Schack D. 2018b. Evaluation of two main RNA-seq approaches for gene quantification in clinical RNA sequencing: polyA+ selection versus rRNA depletion. Sci Rep 8: 4781 .

Zhao W, He X, Hoadley KA, Parker JS, Hayes D, Perou CM. 2014. Comparison of RNA-Seq by poly (A) capture, ribosomal RNA depletion, and DNA microarray for expression profiling. BMC Genomics 15: 419 .

\section{SUPPLEMENTAL MATERIAL (Tables and Files)}

Supplemental Table S1. Clinico-pathological characteristics and recurrence status of the prostate specimens used for the total stranded RNA-sequencing (PAIR, Discovery Set). 
Supplemental Table S2. DE-kupl contigs, PCA3 and housekeeping protein-coding genes for RNA expression measurements by the NanoString nCounter assay.

Supplemental Table S3. Clinico-pathological characteristics, risk classification and recurrence status of the prostate specimens used in NanoString (PAIR, Selection Set).

Supplemental Table S4. PCA3 and DE-kupl contigs expression measurements by the NanoString nCounter assay in 144 specimens of the PAIR cohort (Selection Set). Normalized expression of each probe was calculated as a ratio of the raw value to the mean expression of three housekeeping genes (GPATCH3, ZNF2, ZNF346).

Supplemental Table S5. Mean and Fold Change of expression of PCA3 and DE-kupl contigs in prostate normal and tumor specimens measured by NanoString across 144 prostate specimens of the Selection Set.

Supplemental Table S6. PCA3 and DE-kupl contigs expression quantification assessed by the total stranded RNA-seq in 24 prostate specimens from the PAIR cohort (Discovery Set).

Supplemental Table S7. Clinico-pathological characteristics and recurrence status of the prostate specimens from TCGA-PRAD cohort (Validation Set).

Supplemental Table S8. PCA3 and DE-kupl contigs quantification of expression assessed by the poly(A)+ unstranded RNA-seq in 557 prostate specimens from the TCGA-PRAD cohort (Validation Set).

Supplemental Table S9. Mean and Fold Change expression of PCA3, DE-kupl contigs and housekeeping genes in low-risk (LR) and high-risk (HR) tumors and recurrence negative (NO) and positive (YES) specimens of the PAIR cohort (Selection Set). 